\title{
FINDING REAL RATIONAL WEIGHTING FUNCTIONS FOR ROBUST CONTROL ANALYSIS AND DESIGN; CONNECTIONS TO BOUNDARY INTERPOLATION AND CIRCUIT SYNTHESIS*
}

\author{
ROBERT R. BITMEAD ${ }^{\dagger}$
}

\begin{abstract}
We consider the problem of generating a matrix transfer function with prescribed strict bistability, biproperness, and positivity properties from a sequence of positive definite sample values at points on the frequency axis. We show how this arises in problems from multi-input/multioutput control analysis and design. This is a matrix interpolation problem with additional conditions, both on the nature of the data and the function class of the interpolant solution. The main result is to establish that it is always possible to find a solution to this problem, which has import for the validity of using sampled experimental data in determining non-conservative multi-input/multioutput stability margins via weighting functions. The full class of solutions in presented. The methods of the paper make strong contact with matrix Nevanlinna-Pick interpolation theory and with circuit theory, notably the methods of multi-port circuit synthesis.
\end{abstract}

1. Introduction. The problem addressed in this paper is to find a matrix transfer function, $W(z)$, of a complex variable $z$ which interpolates given positive definite values, $\left\{W_{k}\right\}$, for a sequence of pure-imaginary data points $\left\{z_{k}=j \omega_{k}: k=\right.$ $1, \ldots, N\}$. The conditions on $W(z)$ are that it should be strictly stable (all poles in $\mathbb{R e}[z]<-\alpha<0)$, possess an inverse which is also strictly stable, have both limits $W(\infty)$ and $W^{-1}(\infty)$ positive definite, have positive definite real part for all $z=j \omega$, and interpolate $W\left(z_{k}\right)=W_{k}$. The requirement to find such a matrix interpolant stems from a problem in robust stability, presented in Section 2, where the data $\left\{z_{k}, W_{k}: k=1, \ldots, N\right\}$ arise from the experimental determination of the (generalized) stability margin of a multi-input/multi-output (MIMO) plant-controller closed loop. Then $W(z)$ represents the matrix weighting function required to determine nonconservative estimates of the margin in the MIMO case. It is interesting to note that, while the interpolation problem is meaningful, this weighting function problem does not possess a scalar (SISO) counterpart because (as will be explained) the weighting functions commute around the loop and then cancel in that case. An equivalent MIMO weighting function calculation is applied in some approaches to $\mu$-synthesis control design [25] and to the development of circuit approximations [15, 24]. The main result of this paper is that a family of such solutions $W(z)$ always exists for this data set. This is important because it establishes that computing sampled positive definite weighting function values at a collection of frequencies always admits the existence of an underlying complete transfer function positive for all frequencies. Thus

* Dedicated to Brian Anderson on the occasion of his 70th birthday.

$\dagger$ Department of Mechanical \& Aerospace Engineering, University of California, San Diego, 9500 Gilman Drive, La Jolla, CA 92093-0411, USA, E-mail: rbitmead@ucsd.edu. 
no incipient problems (akin to phase-wrapping between samples in the SISO case) are introduced by the sampling.

The approach taken is to explore in some detail how this problem may be mapped into the realm of Nevanlinna-Pick interpolation theory. Particularly, the books of Ball, Gohberg and Rodman [7] and Bolotnikov and Dym [11] will be used to present the full set of solutions to the interpolation problem when posed for points $z_{i}$ in the open right half plane. A simple invertible mapping of the complex plane is amenable to convert our interpolation problem on the imaginary axis to a related matrix Nevanlinna-Pick problem. The positivity property of the interpolants then permits the linkage to (strictly) positive real functions from circuit theory. This is exploited in developing the solutions and in demonstrating that these solutions always exist for our data sets. This should be compared to the related but more difficult problem of interpolation of general not-necessarily-positive-definite matrix values with unit matrix transfer functions (i.e. stable, stably invertible and biproper but without positivity) treated in the scalar case by Dorato, Park and Li [19] and by Prasanth [35] in the matrix case, where a solution is not always guaranteed to exist.

Drawing together these ideas from interpolation and circuit theory leads to a brief analysis of the connections between circuit synthesis and interpolation, both in the scalar case and in the matrix case. This is of interest because the scalar problems of interpolation with positive real functions, circuit synthesis via reactance extraction, and interpolation with units, all are linked through the appearance of a deflation algorithm which yields lower-order problems by removing one point of interpolation. In the matrix case, while both interpolation and circuit synthesis have been well developed, it is not apparent how one might search for equivalent deflation methods.

1.1. Connection to the works of Brian D.O. Anderson. Brian Anderson has been a pioneering figure in the fields of Circuit Theory, Control Systems, Signal Processing and many related areas of tangency, intersection and parallel direction (to borrow from Alan Willsky). A major factor in his influence over these very broad domains is his capacity to identify the abstract connections and threads underpinning all of these fields. Thus, he has been central in bringing passivity ideas to control and stability and in porting (pun intended) state-space methods into circuit theory, notably in matrix $n$-port analysis and synthesis. His skill is not just in spotting the connections but in using them to bring into play sophisticated machinery, which helps to demystify the subject and empower the user. This is nowhere more apparent than in his work with John Moore in Kalman Filtering. As a student of Brian from 1976 in Newcastle to the present day, I arrived after his heyday in circuit synthesis. Although, my first paper with him was on Lossless Positive Real $(\mathcal{L P R})$ matrix transfer functions [10]. We have enjoyed joint and parallel publications mostly in control, signal processing and estimation since then. I was drawn to Brian's chapter [2] tying the 
Brune Synthesis, formulated by Otto Brune in 1931 [13] and "to whom we owe much in the development of synthesis procedures" to quote Guillemin [23], to the solution of difficult Riccati equation problems in control using the methods of Hamiltonian matrices, spectral factorization and the synthesis approach of deflation. This wideranging trawl across disciplines is indicative of the scope of his knowledge and impact. Accordingly, I have chosen to seek to knot together the requirements of a problem arising in robust control based on experimental data - a subject of Brian's current interest [16] - matrix interpolation theory, and the methods of circuit synthesis, with their genesis ten years before Brian's own appearance.

2. Robust control and the weighting matrix problem statement. Robust stability analysis for MIMO control systems proceeds by building on SISO systems theory concepts of margins associated with; gain, phase, modulus, or delay. A quantified theory of robust stabilization and performance in the face of uncertainty $[46,37,20]$ associates scalar metrics of weighted matrix transfer functions. These metrics capture, in a scalar form, nominal design properties and known or presumed properties of the uncertainty of the system. An archetypal scalar robust stability calculation of this form is the generalized stability margin and $\nu$-gap metric of Vinnicombe [39]. Another example is $\mu$-synthesis and robust performance [46].

In order to ameliorate the conservatism and signal unit selection issues of deriving scalar measures from MIMO designs, weighting function matrices which scale the signal magnitudes may be inserted into the calculations as illustrated in Figure 2.1. Evidently, the two loops are identical and possess the same stability and

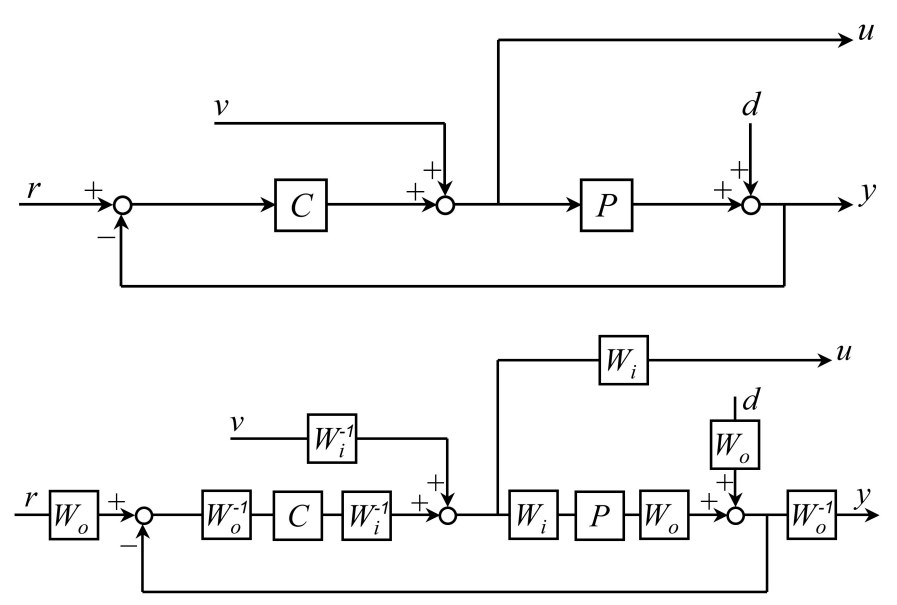

FIG. 2.1. Two equivalent MIMO feedback loops with identical stability and internal stability properties, provided weighting transfer function matrices, $W_{i}$ and $W_{o}$, are stable and stably invertible.

internal stability properties provided the input and output scaling/weighting matrix 
transfer functions, $W_{i}(z)$ and $W_{o}(z)$ respectively, are stable and stably invertible. This is termed bistability. The analysis in this paper considers the selection of these weighting matrix transfer functions, given the frequency-by-frequency specification of their values and subject to bistability and biproperness, the boundedness of both the transfer function and its inverse as $z$ tends to infinity. The aim is to move from the specification of the pointwise matrix frequency response values to the specification of the full bistable biproper transfer function. We shall follow [30] for this issue with the generalized stability margin. For $\mu$-synthesis, an equivalent problem formulation arises, see [25].

The genesis of the problem for the author lies in the certification process for aircraft jet engine-controller pairs, where (often a new) engine is tested with its (new) controller with the intention of verifying that the couple operates sufficiently robustly to accommodate (future) fleet variability due to manufacturing, wear-and-tear, refurbishment, operating regime, etc. of engines yet to be. Normally, for SISO systems, this is performed using specifications on the gain and phase margins measured experimentally on the engine-controller combination [4]. The measurement is performed using frequency response analysis from a closed-loop experiment with broadband excitation. In the MIMO case, the replacement for the gain or phase margin is not immediately apparent, although we advance a natural candidate immediately below. What does remain is the dependence of engine-controller certification on the experimental measurement of closed-loop (matrix) frequency response values taken at frequencies sampled from the continuum of possible values. The introduction of frequency-by-frequency optimized weighting matrices dramatically reduces the conservatism of the MIMO margin calculation, in some examples by as much as four orders of magnitude, yielding workable values. This should be borne in mind as the presentation develops, since it provides contact with the practical needs underpinning the theoretical analysis.

The generalized sensitivity function of MIMO plant-controller pair $(P, C)$ is defined as

$$
T(P, C, z)=\left[\begin{array}{cc}
P(I+C P)^{-1} C & P(I+C P)^{-1} \\
(I+C P)^{-1} C & (I+C P)^{-1}
\end{array}\right] .
$$

For an $m \times p$ plant $P(z)$ and corresponding $p \times m$ controller $C(z), T(P, C, z)$ is an $(m+p) \times(m+p)$ transfer function matrix. When scaling is introduced via $W_{i}(z)$ $(p \times p)$ and $W_{o}(z)(m \times m)$, an altered generalized sensitivity function is produced;

$$
T\left(W_{o} P W_{i}, W_{i}^{-1} C W_{o}^{-1}, z\right)=\left[\begin{array}{cc}
W_{o} & 0 \\
0 & W_{i}^{-1}
\end{array}\right] T(P, C, z)\left[\begin{array}{cc}
W_{o}^{-1} & 0 \\
0 & W_{i}
\end{array}\right],
$$

The frequency-dependent generalized stability margin for the pair $(P, C)$ is a scalar 
and is defined by

$$
b_{P, C}(\omega)= \begin{cases}{\left[\sigma_{\max }(T(P, C, j \omega))\right]^{-1},} & \text { if }(P, C) \text { is stable } \\ 0, & \text { else. }\end{cases}
$$

and the generalized stability margin of $(P, C)$ is then defined by $b_{P, C}=\inf _{\omega} b_{P, C}(\omega)$. The margin $b_{P, C}(\omega) \in[0,1]$, with higher margins implying greater robustness. We note that for MIMO systems in general, but not for SISO systems,

$$
b_{P, C}(\omega) \neq b_{W_{o} P W_{i}, W_{i}^{-1} C W_{o}^{-1}}(\omega) .
$$

The problem considered in [30] is to maximize $b_{W_{o} P W_{i}, W_{i}^{-1} C W_{o}^{-1}}(\omega)$, the frequency-dependent margin, in order to profit, if possible, from its lower conservatism versus $b_{P, C}(\omega)$ and $b_{P, C}$. This is performed frequency by frequency relying on the following result from [30], which commences from the matrix frequency response sample, $T\left(P, C, j \omega_{k}\right)$, of the generalized sensitivity function matrix.

TheOrem $2.1([30])$. At a fixed frequency, $\omega_{k}$, consider positive definite hermitian matrices $W_{i}$ and $W_{o}$ with $X_{o}=W_{o}^{*} W_{o}$ and $Y_{i}=\left(W_{i} W_{i}^{*}\right)^{-1}$. If a solution $\left(X_{o}, Y_{i}\right)$ exists of the following Linear Matrix Inequality (LMI),

$$
\begin{array}{ll}
\text { minimize } & \gamma_{1}^{2} \\
\text { subject to } & \gamma_{1}^{2}\left[\begin{array}{cc}
X_{o} & 0 \\
0 & Y_{i}
\end{array}\right]-T\left(P, C, j \omega_{k}\right)^{*}\left[\begin{array}{cc}
X_{o} & 0 \\
0 & Y_{i}
\end{array}\right] T\left(P, C, j \omega_{k}\right)>0, \\
& X_{o}>0, \quad Y_{i}>0,
\end{array}
$$

with achieved objective value $\gamma_{1}$, then the scaled $b_{P, C}$ at $\omega_{k}$ is bounded below by $\gamma_{1}^{-1}$

$$
b_{W_{o} P W_{i}, W_{i}^{-1} C W_{o}^{-1}}\left(\omega_{k}\right)>\gamma_{1}^{-1} \text {. }
$$

The optimization in (2.4) can be performed iteratively using bisection on $\gamma_{1}^{2}$ by solving feasibility problems as in [12]. This positions the problem of weighting function value selection as a generalized eigenvalue problem to be solved at each sample frequency value.

For a sequence of sampled frequency response values $\left\{T\left(P, C, j \omega_{k}\right) ; k=1,2, \ldots\right.$, $N\}$, the solution of LMI (2.4) yields a sequence of positive definite solutions, $\left\{X_{o}\left(\omega_{k}\right)\right.$, $\left.Y_{i}\left(\omega_{k}\right)\right\}$, and their corresponding sequence of weighting matrix values, $\left\{W_{o}\left(j \omega_{k}\right)\right.$, $\left.W_{i}\left(j \omega_{k}\right)\right\}$ defined to be the positive definite square roots of $X_{o}$ and $Y_{i}^{-1}$ respectively. We pose the following problem associated with the application of these sampled weighting matrices.

\section{Problem statement W:}

Given a sequence of distinct positive frequencies, $\left\{\omega_{k} ; k=1,2, \ldots\right.$, $N\}$, and a corresponding sequence of positive definite square weighting matrix samples, $\left\{W_{k}=W\left(j \omega_{k}\right)\right\}$, satisfying

$$
0<\underline{W} \leq W_{k} \leq \bar{W}<\infty I,
$$


prove that there always exists a matrix transfer function $W(z)$ satisfying the following conditions.

interpolation: $W\left(j \omega_{k}\right)=W_{k}$ for each $k=1, \ldots, N$.

(strict) bistability: $W(z)$ and its inverse, $W(z)^{-1}$, are analytic in the half-plane $\mathbb{R} e[z]>-\alpha$ for some $\alpha>0$.

(strict) positivity: $W(j \omega)>0$ and $W^{-1}(j \omega)>0$ for all real $\omega$. biproperness: $W(\infty)>0$ and $W(\infty)^{-1}>0$.

The problem statement is clearly one of finding bistable biproper matrix transfer functions, $W_{i}(z)$ and $W_{o}(z)$, which interpolate the positive definite sample values on the imaginary axis. We remark that the positive quantity $\alpha$ in the bistability condition above is related to the smoothness of the frequency response $W(j \omega)$ between the sample frequency values. Next we move on to present some classical and more recent results from interpolation theory before drawing some parallels to circuit theory and the properties of positive real functions in circuit synthesis. To foreshadow this discussion, we remark that strictly positive real $(\mathcal{S P R})$ transfer functions satisfy the positivity and bistability properties of the problem statement and that these transfer functions represent the driving-point immittances of strictly passive linear circuits. Connections between passive circuit synthesis and interpolation problems have been known at least since Belevitch $[9,43]$.

3. Nevanlinna-Pick interpolation background. The weighting matrix transfer function problem enunciated in Section 2 is one of matrix interpolation in the complex plane. The results in this field consider a number of differing function classes and two distinct domains of interpolation. Denote by $\mathbb{D}$ the open unit disk $|z|<1$ and by $\Pi^{+}$the open right half plane $\mathbb{R} e[z]>0$. Further, denote the $p \times p$ matrix-valued function $F(z)$ of complex variable $z$ as belonging to;

Class $\mathcal{C}$ (Carathéodory): if $F(z)$ is analytic and $\mathbb{H} e[F(z)] \geq 0$ for all $z$ in the open unit disk $\mathbb{D}$,

Class $\mathcal{S}$ (Schur): if $F(z)$ is analytic and contractive, i.e. $\sigma_{\max }(F(z)) \leq 1$, for all $z$ in the open unit disk $\mathbb{D}$,

Class $\mathcal{B R}$ (Bounded real): if $F(z)$ satisfies $F(z)=\bar{F}(\bar{z})$ and is analytic and contractive for all $z$ in the open right half plane $\Pi^{+}, \mathbb{R} e[z] \geq 0$.

Class $\mathcal{P} \mathcal{R}$ (Positive real): if $F(z)$ satisfies $F(z)=\bar{F}(\bar{z})$ and is analytic and $\mathbb{H} e[F(z)] \geq 0$ for all $z$ in the open right half complex plane $\Pi^{+}, \mathbb{R} e[z] \geq 0$.

Class $\mathcal{S P \mathcal { R }}$ (Strictly positive real): if $F(z-\alpha)$ is class $\mathcal{P} \mathcal{R}$ for some real $\alpha>0$.

Here the overbar $\left(^{-}\right)$denotes complex conjugation, the superscript star $\left(^{\star}\right)$ indicates conjugate transpose, and $\mathbb{H} e[X]$ represents the hermitian part, $\frac{1}{2}\left(X+X^{\star}\right)$. When applied to a function, complex conjugation is applied to the coefficients of the function and not to its independent variable. A function $F$ belongs to class $\mathcal{C}$ if and only if $G(z)=\left[I_{p}-F(z)\right]\left[I_{p}+F(z)\right]^{-1}$ belongs to class $\mathcal{S}$. Function $F(z)$, real-valued for 
real $z$, belongs to class $\mathcal{C}$ if and only if $H(s)=F\left(\frac{z-1}{z+1}\right)$ is class $\mathcal{P} \mathcal{R}$. Function $F(z)$ belongs to $\mathcal{P R}$ if and only if $G(z)=\left[I_{p}-F(z)\right]\left[I_{p}+F(z)\right]^{-1}$ belongs to class $\mathcal{B R}$. Our focus will be on the classes $\mathcal{P} \mathcal{R}$ and $\mathcal{S P \mathcal { R }}$ and on the domain $\Pi^{+}$, the open right half plane, and its closure $\overline{\Pi^{+}}$.

Positive real functions are well known from circuit theory associated with the immittances of circuits composed of passive elements and from the study of passive linear systems. This function class $\mathcal{P} \mathcal{R}$ almost captures the requirements of Problem W, except that $\mathcal{P} \mathcal{R}$-functions may possess poles and zeros on the imaginary axis provided these are simple and provided the residue at the imaginary pole is nonnegative definite. Similarly, $\mathcal{P} \mathcal{R}$-functions may possess a simple zero or pole (with nonnegative residue) at infinity, such as occurs with capacitive or inductive impedances respectively. The presence of infinite or pure imaginary poles or zeros would violate both the strict positivity and the bistability conditions. Accordingly, the interest is in determining interpolants $W(z)$ in class $\mathcal{S P R}$ with biproper behavior at infinity.

Recall [3] that if $W \in \mathcal{P} \mathcal{R}$ then $W^{-1} \in \mathcal{P} \mathcal{R}$. Accordingly, if $W \in \mathcal{S P} \mathcal{R}$ then $W^{-1} \in \mathcal{S P R}$ and consequently $W$ and $W^{-1}$ are then strictly positive for finite $z \in \Pi^{+}$. If further $W(\infty)$ is finite and positive definite, and thus so is $W^{-1}(\infty)$, then both $W$ and $W^{-1}$ are bounded on the imaginary axis. This leads to the following result.

Lemмa 3.1. If $F(z) \in \mathcal{S P R}$ satisfies the interpolation condition of Problem (W) and has $F(\infty)$ finite and invertible, then it also satisfies the bistability, biproperness, and strict positivity conditions and, thus, $F(z)$ is a solution of $(\mathbb{W}$.

We shall rely on this lemma to focus attention firstly on achieving the interpolation in class $\mathcal{P} \mathcal{R}$ and then in class $\mathcal{S P \mathcal { R }}$ - a problem associated with interpolation by units in $\mathcal{R} \mathcal{H}_{\infty}[19,35]$ - and then establish a simple procedure from [35] for ensuring the biproperness of the interpolant. We begin by first considering the interpolation in class $\mathcal{P} \mathcal{R}$, which is well studied.

3.1. Scalar Nevanlinna-Pick Interpolation. The Pick problem $[1,33]$ is to determine a class $\mathcal{C}$ function, $f(z)$, which interpolates a sequence of complex values, $\left\{w_{1}, w_{2}, \ldots, w_{N}\right\}$, at specific points, $\left\{z_{1}, z_{2}, \ldots, z_{N}\right\}$ inside $\mathbb{D}$. The condition for the solubility of this problem is the positive semi-definiteness of the Pick Matrix,

$$
P_{i, j}=\frac{w_{i}+\bar{w}_{j}}{1-z_{i} \bar{z}_{j}}
$$

Moreover, the solution is unique if this matrix possesses zero determinant. Nevanlinna [27] considered a similar problem and provided a characterization of the complete set of solutions including the problem with a countably infinite set of interpolation points [28]. This problem is known as the Nevanlinna-Pick Interpolation Problem and often is alternatively stated in terms of the function $f(z)$ belonging to class $\mathcal{S}$ for which case the associated Pick matrix is then given by

$$
\bar{P}_{i, j}=\frac{1-w_{i} \bar{w}_{j}}{1-z_{i} \bar{z}_{j}} .
$$


For scalar (one-port) circuit problems, Youla and Saito [43] develop positive real impedance synthesis based on reformulation of Nevanlinna-Pick interpolation to address interpolation and analyticity in $\Pi^{+}$, the open right-half complex plane, and nonnegativity of the matrix

$$
A_{i . j}=\frac{w_{i}+\bar{w}_{j}}{z_{i}+\bar{z}_{j}}
$$

We shall return to the connections between these circuit synthesis problems and the approach to solution of Nevanlinna-Pick interpolation problems later in Section 5.

3.2. Extension to matrix-valued functions. The extension of NevanlinnaPick interpolation problems to include matrix-valued functions over the complex plane is usually attributed to work by Fedčina [21, 22], although here we shall follow the development of [7]. We shall present these results in some detail for completeness. That is, we shall consider two-sided tangential interpolation - an encoding of matrix interpolation to be clarified shortly - but restrict analysis to the domain $\Pi^{+}$and interpolant class $\mathcal{P} \mathcal{R}$ because this allows us to present the results in their generality. The extension to domain $\mathbb{D}$ and class $\mathcal{S}$ is available in [7]. Subsequently, we shall specialize just to left interpolation of square matrix values, since this suffices for our purposes, and then restrict this further to bounded positive definite interpolation values.

The expression of the matrix generalization of Nevanlinna-Pick interpolation involves a data set of seven matrices $\omega=\left(C_{+}, C_{-}, A_{\pi} ; A_{\zeta}, B_{+}, B_{-} ; S\right)$, with the subsets of matrices; $\left(C_{+}, C_{-}, A_{\pi}\right)$ associated with right tangential interpolation, $\left(B_{+}, B_{-}, A_{\zeta}\right)$ associated with left tangential interpolation, $\left(S, B_{+}, C_{-}, A_{\pi}, A_{\zeta}\right)$ associated with twosided interpolation. The eigenvalues of $A_{\pi}$ and $A_{\zeta}$ lie in $\Pi^{+}$. It is assumed that the set $\omega$ is admissible.

Interpolation data admissibility

The data set $\omega=\left(C_{+}, C_{-}, A_{\pi} ; A_{\zeta}, B_{+}, B_{-} ; S\right)$ is admissible if;

i. $C_{+}, C_{-}, A_{\pi}, A_{\zeta}, B_{+}, B_{-}, S$ have dimensions $p \times n_{\pi}, m \times n_{\pi}, n_{\pi} \times n_{\pi}, n_{\zeta} \times n_{\zeta}$, $n_{\zeta} \times p, n_{\zeta} \times m, n_{\zeta} \times n_{\pi}$, respectively,

ii. $\left(C_{-}, A_{\pi}\right)$ is observable and $\left(A_{\zeta}, B_{+}\right)$is controllable,

iii. the eigenvalues of $A_{\pi}$ and of $A_{\zeta}$ all lie in $\Pi^{+}$, and

iv. $S$ satisfies the Sylvester equation

$$
S A_{\pi}-A_{\zeta} S=B_{+} C_{+}-B_{-} C_{-} .
$$

The generalized $(\mathcal{P} \mathcal{R})$ Nevanlinna-Pick interpolation problem is to find a matrix function of class $\mathcal{P} \mathcal{R}$, i.e.

$$
F(z) \text { is analytic and satisfies } \mathbb{H} e[F(z)] \geq 0 \text { for } z \text { in } \Pi^{+},
$$


such that

$$
\begin{aligned}
& \sum_{z \in \Pi^{+}} \operatorname{Res}\left\{\left(z I-A_{\zeta}\right)^{-1} B_{+} F(z)\right\}=-B_{-}, \\
& \sum_{z \in \Pi^{+}} \operatorname{Res}\left\{F(z) C_{-}\left(z I-A_{\pi}\right)^{-1}\right\}=C_{+}, \\
& \sum_{z \in \Pi^{+}} \operatorname{Res}\left\{\left(z I-A_{\zeta}\right)^{-1} B_{+} F(z) C_{-}\left(z I-A_{\pi}\right)^{-1}\right\}=S .
\end{aligned}
$$

Here the matries $A_{\zeta}$ and $A_{\pi}$ have eigenvalues in $\Pi^{+}$and the residue formula is used to capture the interpolation conditions such as $B_{+, i} F\left(z_{i}\right)=-B_{-, i}$, for specific submatrices of $B_{+}$and $B_{-}$. In the later analysis, left interpolation will suffice and we shall take

$$
A_{\zeta}=\left(\begin{array}{cccc}
z_{1} I_{p} & 0_{p} & \ldots & 0_{p} \\
0 & z_{2} I_{p} & \ldots & 0_{p} \\
\vdots & & \ddots & \vdots \\
0_{p} & 0_{p} & \ldots & z_{N} I_{p}
\end{array}\right), \quad B_{+}=\left(\begin{array}{c}
I_{p} \\
I_{p} \\
\vdots \\
I_{p}
\end{array}\right), \quad B_{-}=-\left(\begin{array}{c}
W_{1} \\
W_{2} \\
\vdots \\
W_{N}
\end{array}\right),
$$

together with vacuous matrices

$$
C_{-}=C_{+}=A_{\pi}=S=() .
$$

The admissibility of the data $\left(A_{\zeta}, B_{+}, B_{-}\right)$then reduces to the correct dimension of the matrices, the controllability of $\left(A_{\zeta}, B_{+}\right)$, and the eigenvalues of $A_{\zeta}$ lying in $\Pi^{+}$, each of which conditions is evidently satisfied by the choice in (3.8) for $F\left(z_{k}\right)=W_{k}$ with $z_{k} \in \Pi^{+}$.

Ball, Gohberg and Rodman provide the following solution to this problem.

Theorem 3.2 ([7] Theorem 22.2.2). Let $\omega=\left(C_{+}, C_{-}, A_{\pi} ; A_{\zeta}, B_{+}, B_{-} ; S\right)$ be an admissible data set where $C_{-}$and $C_{+}$have $p$ rows and $B_{+}$and $B_{-}$have $p$ columns. Define matrices $\Lambda_{1}$ sand $\Lambda_{2}$ as the unique solutions of the respective Lyapunov equations

$$
\Lambda_{1} A_{\pi}+A_{\pi}^{\star} \Lambda_{1}=C_{+}^{\star} C_{-}+C_{-}^{\star} C_{+},
$$

and

$$
\Lambda_{2} A_{\zeta}^{\star}+A_{\zeta} \Lambda_{2}=-B_{+} B_{-}^{\star}-B_{-} B_{+}^{\star},
$$

and set

$$
\Lambda=\left(\begin{array}{cc}
\Lambda_{1} & S^{\star} \\
S & \Lambda_{2}
\end{array}\right)
$$

Then there exists a rational square matrix function $F(z)$ analytic with positive definite hermitian part on the closed right half plane $\overline{\Pi^{+}}$which satisfies the interpolation 
conditions (3.5-3.7) if and only if the matrix $\Lambda$ is positive definite. Moreover, in this case the set of all such matrix functions $F(z)$ is given by

$$
F(z)=\left(\Theta_{11}(z) G(z)+\Theta_{12}(z)\right)\left(\Theta_{21}(z) G(z)+\Theta_{22}(z)\right)^{-1},
$$

where $G(z)$ is an arbitrary rational $p \times p$ matrix function analytic and with positive definite hermitian part on the closed right half plane $\overline{\Pi^{+}}$and

$$
\begin{aligned}
\Theta(z) & =\left(\begin{array}{ll}
\Theta_{11}(z) & \Theta_{12}(z) \\
\Theta_{21}(z) & \Theta_{22}(z)
\end{array}\right), \\
& =I_{2 p}+\left(\begin{array}{ll}
C_{+} & B_{-}^{\star} \\
C_{-} & B_{+}^{\star}
\end{array}\right)\left(\begin{array}{cc}
\left.z I-A_{\pi}\right)^{-1} & 0 \\
0 & \left(z I+A_{\zeta}^{\star}\right)^{-1}
\end{array}\right) \Lambda^{-1}\left(\begin{array}{ll}
C_{-}^{\star} & C_{+}^{\star} \\
B_{+} & B_{-}
\end{array}\right) .
\end{aligned}
$$

The conditions for existence of solutions for the left interpolation data (3.8) follow simply from Theorem 3.2 .

Corollary 3.3. Let the left interpolation data be given by (3.8) with $z_{i} \in \Pi^{+}$for $i=1, \ldots, N$. Then there exists a solution for $F(z)$ analytic and obeying $\mathbb{H} e[F(z)]>0$ on $\overline{\Pi^{+}}$and satisfying $F\left(z_{i}\right)=W_{i}$ if and only if the matrix $\Lambda$ with $(i, j)$ th block

$$
\Lambda_{i, j}=\frac{W_{i}+W_{j}^{\star}}{z_{i}+\bar{z}_{j}}
$$

is positive definite. In this case, the set of solutions is given by

$$
F(z)=\left(\Theta_{11}(z) G(z)+\Theta_{12}(z)\right)\left(\Theta_{21}(z) G(z)+\Theta_{22}(z)\right)^{-1},
$$

where $G(z)$ is an arbitrary rational $p \times p$ matrix function analytic and obeying $\mathbb{H} e[G(z)]$ $>0$ on $\overline{\Pi^{+}}$and

$\Theta(z)=\left(\begin{array}{cc}\Theta_{11}(z) & \Theta_{12}(z) \\ \Theta_{21}(z) & \Theta_{22}(z)\end{array}\right)$

$$
=I_{2 p}+\left(\begin{array}{c}
B_{-}^{\star} \\
B_{+}^{\star}
\end{array}\right)\left(z I+A_{\zeta}^{\star}\right)^{-1} \Lambda^{-1}\left(\begin{array}{ll}
B_{+} & B_{-}
\end{array}\right),
$$

$$
=I_{2 p}+\left(\begin{array}{cccc}
-W_{1}^{\star} & -W_{2}^{\star} & \ldots & -W_{N}^{\star} \\
I_{p} & I_{p} & \ldots & I_{p}
\end{array}\right) \text { blkdiag }\left[\left(z+\bar{z}_{i}\right)^{-1} I_{p}\right] \Lambda^{-1}\left(\begin{array}{cc}
I_{p} & -W_{1} \\
I_{p} & -W_{2} \\
\vdots & \vdots \\
I_{p} & -W_{N}
\end{array}\right)
$$

The proof of this result follows directly by showing by substitution that $\Lambda$ in (3.13) satisfies the sole Lyapunov equation (3.10) associated with (3.8),

$$
\Lambda A_{\zeta}^{\star}+A_{\zeta} \Lambda=-B_{+} B_{-}^{\star}-B_{-} B_{+}^{\star} .
$$


Delsarte, Genin and Kamp [17] develop a condition equivalent to $\Lambda>0$ (in the domain $\mathbb{D}$, class $\mathcal{S}$ case) and show that a solution via Nevanlinna's algorithm is related to the Cholesky decomposition of matrix $\Lambda$. An iterative solution then evolves by using this Cholesky decomposition to compute $\Lambda^{-1}$.

We remark, since it will arise shortly, that when the condition $\mathbb{H} e[G]>0$ is relaxed to $\mathbb{H} e[G] \geq 0$ on $\overline{\Pi^{+}}$, but we still have $\Lambda>0$, then the analyticity of $F(z)$ is still guaranteed on $\Pi^{+}$, as is the nonnegativity and interpolation. Indeed, taking $G=0$ is one such circumstance. The only-if part of the statement then becomes more difficult.

4. Main result. The forward interpolation problem, i.e. that of moving from the interpolation points and matrix values $\left\{z_{k}, W_{k}\right\}$ in $\Pi^{+}$to a class $\mathcal{P} \mathcal{R}$ interpolating matrix function, $W(z)$, is covered by the preceding results. The zeros of $W(z)$ or poles of $W^{-1}(z)$ are also implicitly managed by the property that the inverse of a $\mathcal{P R}$ function is also of class $\mathcal{P} \mathcal{R}$, although there is no prohibition on the interpolants possessing either zeros or poles in the immediate neighborhood of the imaginary axis. Further, the behavior at infinity is unspecified. Accordingly, to move further towards the requirements of a solution of Problem (W) will necessitate examination of three modifications to these results, each of which we undertake in this section; movement of the interpolation points to the imaginary axis (the boundary of $\Pi^{+}$), establishment of $\mathcal{S P} \mathcal{R}$ interpolants, and management of the biproperness.

Recent attempts to apply Nevanlinna-Pick interpolation in the passive approximation of microwave components to be used in circuit design [15] illustrate the problem with localizing system zeros. Sampled frequency response data are used as the basis of the interpolation. The larger the number of points used in the interpolation the more erratic the interpolation functions response becomes between the sample points. Further, nulls and the presence of phase wrapping between sample points of the frequency response are evident. The authors apply a convex optimization algorithm to assist in the management of sample points used in the approximation, eventually achieving a smooth, low-order and accurate approximation. Our aim is to establish that it is always possible, for the weighting function data set from Problem $\mathbb{W}$, to find a bistable and biproper solution with a correspondingly smooth frequency response of a biproper $\mathcal{S P \mathcal { R }}$ function. In doing so, the positivity of the weighting function samples themselves will play an important role in guaranteeing the existence of suitable interpolants.

4.1. Extension to boundary interpolation and $\mathcal{S P} \mathcal{R}$. Evidently, there is a difficulty with the matrix $\Lambda$ of (3.13) when the interpolation points $z_{i}$ are permitted to lie on the imaginary axis in the $z$ complex plane. However, it is also apparent that, by taking limits as the points $z_{i}$ tend towards the imaginary axis, the positivity condition on $\Lambda$ becomes trivially satisfied sufficiently close to the axis. [Recall that 
necessarily the interpolation data values must satisfy $W_{i}+W_{i}^{\star}>0$ for class $\mathcal{P} \mathcal{R}$ in order for a solution to exist and that, further, our weighting function data values from Problem @ are positive definite by construction.] Thus, the detailed solution really only becomes of interest when these strict inequalities fail on the boundary. This problem is known as Löwner interpolation and has been considered by a number of researchers [6, 36]. The results of [7] in matrix Nevanlinna-Pick interpolation are extended to boundary problems in Chapter 21 of that book. Also, Bolotnikov and Dym [11] devote a chapter to the Nevanlinna-Pick boundary problem for the class $\mathcal{S}$ problem. As expected, the results carry through subject to regularity conditions arising from the points on the boundary or frequency axis.

However, because we are interested only in the case of positive definite interpolation values on the imaginary axis, we can get by with modifying the earlier results. The approach we shall take with Problem (W) and the boundary interpolation of weighting function matrices will be to use a simple invertible linear mapping of the complex plane and then to rely on the earlier results of generalized Nevanlinna-Pick interpolation. Indeed, this is countenanced as an approach to well-behaved boundary interpolation problems in $[7,11,43]$. We shall rely on the strict positivity of the frequency samples $W_{k} \geq \underline{W}>0$ to make this simple modification to the interpolation in the right half plane.

Lemma 4.1. Consider the matrix valued function $F^{\circ}(z)$ analytic in $\Pi^{+}$and interpolating the values $\left\{W_{k}: k=1, \ldots, N\right\}$ at the complex values $\left\{\alpha+j \omega_{k}\right\}$, for $\alpha>0$ and therefore with $\alpha+j \omega \in \Pi^{+}$, and satisfying $\mathbb{H} e\left[F^{\circ}(z)\right] \geq 0$ in $\Pi^{+}$. Then

$$
F(z)=F^{\circ}(z+\alpha)
$$

is analytic and satisfies $\mathbb{H} e[F(z)]>0$ in $\mathbb{R} e[z]>-\alpha+\epsilon$ (for some small $\epsilon$ ) and interpolates $F\left(j \omega_{k}\right)=W_{k}$. Thus, $F(z)$ and $F^{-1}(z)$ are $\mathcal{S P R}$.

THEOREM 4.2. For frequency values $\left\{\omega_{k}\right\}$ and positive definite weighting matrix samples $\left\{W_{k}\right\}$ from Problem $(\mathbb{W}$, there always exists a real positive value $\alpha>0$ such that the matrix $\Lambda$ with $(i, \ell)$ th block

$$
\Lambda_{i, \ell}=\frac{W_{i}+W_{\ell}^{\star}}{2 \alpha+j \omega_{i}-j \omega_{\ell}},
$$

is positive definite.

Thus, by Corollary 3.3 and Lemma 4.1, there exists a matrix function $W(z)$, analytic in $\mathbb{R} e[z]>-\alpha$, obeying $\mathbb{H} e[W(z)]>0$ in $\mathbb{R} e[z]>0$, and interpolating $W\left(j \omega_{k}\right)=W_{k}$.

COROLlary 4.3. If there exists a solution to the weighting function interpolation problem (W), then there exists an hermitian solution to $\mathbf{W}$.

Proof. Take solution $W(z)$ to $\mathbb{W}$, then $\tilde{W}(z)=\frac{1}{2}\left[W(z)+W^{\star}(z)\right]$ is hermitian and interpolates the hermitian values $W_{k}$, has the same hermitian part as $W(z)$, and is analytic in $\mathbb{R} e[z]>0$. 
These results demonstrate the capacity to solve the weighting function forward interpolation problem. That is, given $\left\{\omega_{k}, W_{k}\right\}$ find stable interpolating $W(z)$. They also indicate that, by restricting attention to the $\mathcal{S P} \mathcal{R}$ class, we can simultaneous solve the strict stability properties on $W^{-1}(z)$. What remains is to deal with the behavior at infinity, which we now do.

4.2. Biproperness. The formula (3.14) delivers the complete set of $\mathcal{P} \mathcal{R}$ interpolants. Since a family of solutions exists, it is clear that some solutions (or choices of $G(z)$ ) might provide better properties than others. As we know from Lemma 3.1, if we can find an interpolant in class $\mathcal{S P} \mathcal{R}$ which also satisfies the biproperness condition, then this is a solution to Problem $\mathbb{W}$. We consider below how choices for free variable function $G(z)$ in (3.14) can ensure biproperness of $F(z)$.

4.2.1. A straightforward transcendental solution. If rationality of the solution is not required, then the positivity of the interpolating values together with Nevanlinna-Pick interpolation on shifted $z_{i}$ may be used directly to yield a transcendental interpolant with the property that it is bistable, biproper, and has no zeros closer to the frequency axis than it has poles. However, we are able to manage directly the lower bound on the interpolant using matrix logarithms.

For positive definite matrices $W_{k}$ it is possible to define their matrix logarithm, $\ln W_{k}$, as follows.

Definition For matrix $W>0$ with eigenvalue decomposition $W=U D U^{\star}$ with $U$ unitary and $D=\operatorname{diag}\left[d_{i}\right] d_{i}>0$, we define the logarithm of $W$ as; $\ln W=$ $U \operatorname{diag}\left[\ln \left(d_{i}\right)\right] U^{\star}$.

Note that $\ln W$ has the same dimensions as $W$ and that the matrix exponential is the inverse function of the logarithm, $\exp (\ln W)=W$.

We may now take the interpolation values $W_{k}$ from $(\mathbb{W}$ and replace them by their matrix logarithms, since each value is positive definite. Denote these logarithms,

$$
L_{k}=\ln W_{k} .
$$

Now form the Nevanlinna-Pick interpolant $L(z)$ of the matrix data $\left\{\omega_{k}, L_{k}: k=\right.$ $1, \ldots, N\}$, using a shifting property detailed in the proof to keep the interpolation values positive definite. Denote this $\mathcal{P} \mathcal{R}$ interpolant as $L(z)$.

THEOREM 4.4. Problem (W) always has a solution, satisfying the interpolation, positivity, bistability, and biproperness conditions, of the following form

$$
W(z)=\exp [L(z)]
$$

where $L(z)$ is an interpolant of $\left\{L_{k}=\ln W_{k}\right\}$ at the points $\left\{j \omega_{k}\right\}$ for $k=1, \ldots, N$.

Proof. The matrix $\underline{W}$ from Problem $(\mathbb{W}$ is a lower bound on the interpolation values $W_{k}$. Take any real number $\gamma<\ln \left[\lambda_{\min }(\underline{W})\right]$. Then $e^{-\gamma} W_{k}>I$ for all $k$ and, hence $L_{k}-\gamma I>0$. Using Theorem 4.2 and Corollary 3.3, we may compute a class 
$\mathcal{P R}$ interpolant, $\tilde{L}(z)$, for the data points $\left\{L_{k}-\gamma I\right\}$. Taking $G(z)=0$ in (3.14), we see from allowing $z \rightarrow \infty$ in (3.15) that $\tilde{L}(\infty)=0$. [This is permissible even though $G>0$ is presented in the Theorem, since we shall no longer require strict positivity. See the comment following Corollary 3.3.] Then apply the construction from (4.1) to yield

$$
W(z)=\exp [L(z)]=\exp [\tilde{L}(z)+\gamma I]=\exp (\gamma) \exp [\tilde{L}(z)]
$$

which is analytic and strictly proper in $\mathbb{R} e[z]>-\alpha$. The matrix function $W(z)$ from (4.4), with corresponding

$$
W^{-1}(z)=\exp [-L(z)]=\exp (-\gamma) \exp [-\tilde{L}(z)]
$$

interpolates $\left\{W_{k}\right\}$, is analytic and bounded in $\Pi^{+}$, as is $W^{-1}(z)$. Further, $\mathbb{H} e[W(z)]>$ 0 and $\mathbb{H} e\left[W^{-1}(z)\right]>0$ in $\mathbb{R e} e[z]>-\alpha$ and so this $W(z)$ solves Problem (W).

4.2.2. Rational solutions. The approach in Theorem 4.4 is facile and direct, relying on the positivity properties of the interpolating values. However, the solution is irrational and, hence, unamenable to incorporation into computational packages, which might rely on providing a finite-dimensional state-space realization of the weighting function $W(z)$. Having established the existence of a solution to Problem (W), the next question is to ask whether there also exists a rational solution and to provide guidance to finding it. As stated earlier, the core hardship is that Nevanlinna-Pick interpolation does not directly address the location of the zeros of the interpolants, which conflicts with the bistability requirement. Using Corollary 3.3 we may achieve biproperness while preserving rationality, as was noted in [35].

THEOREM 4.5 (Biproperness). If there exists a solution to the weighting function matrix interpolation problem (W), then there exists a biproper solution to (W).

Proof. Suppose we have a solution $W(z)$ for the interpolation problem. Then, by Corollary 3.3, the matrix $\Lambda$ in (3.13) is positive definite and the complete set of solutions is given by the linear fractional transformation (3.14). Note that, letting $z \rightarrow \infty$, in the definition of the matrix function $\Theta(3.15)$ we have $\Theta_{11}(z) \rightarrow I, \Theta_{22} \rightarrow I$, $\Theta_{12} \rightarrow 0$ and $\Theta_{21} \rightarrow 0$. Thus, from (3.14), $\lim _{z \rightarrow \infty} F(z)=\lim _{z \rightarrow \infty} G(z)$ and $G(z)$ is an arbitrary $\mathcal{P} \mathcal{R}$ function. Choosing biproper $G(z)$ yields a biproper interpolant $W(z)$ from $W^{\circ}(z)$ via (3.14) and (4.1).

4.3. Main theorem. Stable transfer functions in $\mathcal{R} \mathcal{H}_{\infty}$ with stable inverses also in $\mathcal{R} \mathcal{H}_{\infty}$ are known as units. Because of the role played by both units and interpolation in robust control theory $[18,42,46]$, there have been a number of papers focused on interpolation with units as an extension of Nevanlinna-Pick interpolation to include restriction to unit interpolants $[19,26,29,31,32,35]$. However, we are dealing solely with systems satisfying positive definite interpolation values on the imaginary axis. As Lemma 3.1 shows, we may restrict our attention to $\mathcal{S P \mathcal { R }}$ rational interpolants, 
which are a subset of the set of the units in $\mathcal{R H}_{\infty}$. Note that Prasanth [35] shows that all units are products of sequences of $\mathcal{S P R}$ functions, even in the matrix case. We have the following main result which builds in an obvious way on the preceding material.

THEOREM 4.6. There always exists a family of real rational hermitian solutions to Problem W.

The proof of this theorem is constructive.

- Given the sequence of positive frequency values and their corresponding positive definite matrices $\left\{\omega_{k}, W_{k}: k=1, \ldots, N\right\}$, select a real positive value $\alpha$ such that matrix $S$ in (4.2) is positive definite, as is assured possible by Theorem 4.2.

- Using the formulæ of Corollary 3.3 with $G(z)$ an arbitrary biproper $\mathcal{P R}$ function $-G(z)=\rho I$ would do - construct the biproper rational interpolating function $F^{\circ}(z)$ to the data points $\left\{\alpha+j \omega_{k}, W_{k}: k=1, \ldots, N\right\}$ using (3.14).

- Extend this solution to be hermitian, using the construction of Corollary 4.3, yielding $F_{H}^{\circ}(z)$.

- Next shift this solution back to the $\mathcal{S P R}$ biproper rational interpolating function $W(z)=F_{H}^{\circ}(z+\alpha)$.

This $W(z)$ is the required solution to (W).

5. Connections to circuit synthesis. As indicated earlier, $\mathcal{P} \mathcal{R}$ and $\mathcal{S P R}$ functions arise as immittances of passive and strictly passive circuits. Lossless Positive Real $(\mathcal{L P} \mathcal{R})$ transfer function matrices describe the immittances of $p$-port circuits comprised exclusively of lossless elements; capacitors, inductors, transformers, and gyrators. $\mathcal{L P} \mathcal{R}$ transfer functions are also $\mathcal{P} \mathcal{R}$ but have their poles and zeros restricted to the imaginary axis. The problem of passive circuit synthesis is that of commencing with a $\mathcal{P} \mathcal{R}$ transfer function, $Y(z)$, and systematically constructing a circuit composed of passive elements whose $p$-port driving-point immittance is given by $Y(z)$. This is a classical circuit theory problem, which underpins many parts of Linear Systems Theory, since its formal analysis from the 1930s onwards.

Many of the methods of circuit synthesis, such as Brune and Darlington syntheses, concentrate on the extraction of lossless sections and components followed by termination in a single resistive load [23]. Youla's approach [41] unifies much of this for one-ports and develops an algorithm for order-reduction through the successive insertion of lossless two-ports terminated by a resistor. Youla and Saito [43] in their seminal paper tying $\mathcal{P} \mathcal{R}$ (1-port) circuit synthesis to Nevanlinna-Pick interpolation also focus on the generation of $\mathcal{P} \mathcal{R}$ circuits whose impedances interpolate specific values at points $z_{k} \in \Pi^{+}$. The positivity of the appropriate Pick matrix from (3.3) naturally arises as the condition for this, but the synthesis approach concentrates on the calculation of so-called Foster (i.e. $\mathcal{L P} \mathcal{R}$ ) circuits which achieve the interpolation. 
The emphasis on lossless realizations derives from the desire to minimize the noise figures of the circuits by managing the number of resistors.

The work of Youla and Saito was preceded by studies of the problem of interpolation on the real frequency axis, which connects strongly with the weighting function interpolation statement (W) in that it involves the synthesis of a $\mathcal{P} \mathcal{R}$ circuit whose impedance matches specified values at given points on the imaginary axis - real frequencies. The approaches were iterative and reminiscent of Lagrange interpolation [14], where a common annihilating polynomial possessing zeros at each interpolation point has one zero cancelled to achieve each interpolation. While Youla in [41] floats the idea of using his methods for interpolation in $\Pi^{+}$, it appears that Wohlers [40] was the first to refine the method to use as a frequency response interpolation process and showed how this yielded a $\mathcal{P} \mathcal{R}$ interpolant. Smilen's paper [38] and its successors due to Zeheb and Lempel [45] and Beccari [8] develop these ideas of the Youla construction further in producing transformerless circuit sections yielding syntheses for impedances interpolating frequency response values in a very systematic procedure. Particularly, the work of Zeheb and Lempel [45] yields a lossless two-port terminated by a single resistor. Their approach is quite elegant and connected to both Darlington synthesis and Lagrange interpolation, since it results in the construction of a lossless two-port consisting of multiple parallel branches of cascaded simple lossless two-ports with carefully located poles and zeros, effectively constructing a common annihilator polynomial, followed by $L$ and $C$ components, which determine the interpolation value associated with each branch, and terminated in a single resistor. It should be remarked that some of these synthesis approaches, while elegant, can yield highly non-minimal circuits in terms of the number of reactive elements used. It is also worthy of note that these frequency response interpolation problems always admit a solution; the Pick matrix does not arise as part of the analysis.

From the perspective of Problem (W) however, the emphasis of these synthesis methods on lossless interpolation fails to recognize the requirements for strict positivity, bistability and biproperness. Further, it is not always apparent how one might move from the scalar one-port synthesis to a matrix $p$-port approach, since, for example, the interpolation values $W_{k}$ may no longer be ordered in the matrix case. In their paper [19] on the interpolation in $\Pi^{+}$by scalar transfer functions which are units in $\mathcal{R H}_{\infty}$, Dorato, Park and Li demonstrate that the Youla and Saito recursion preserves not just the $\mathcal{P} \mathcal{R}$ property of successive cascaded sections but also preserves their $\mathcal{S P \mathcal { R }}$ properties. This is then applied to develop an algorithm for unit interpolation, noting that biproper $\mathcal{S P \mathcal { R }}$ functions are a (strict) subset of the set of units. Prasanth's [35] extension of the interpolation problem with units from the scalar case to the matrix case relies on Theorem 22.2.2 from [7] as above. He also explores interpolation with biproper $\mathcal{S P \mathcal { R }}$ functions and shows inter alia that all units in $\mathcal{R} \mathcal{H}_{\infty}^{p \times p}$ can be written as the product of $\mathcal{S P \mathcal { R }}$ factors. However, the iterative nature of the 
Youla and Saito approach is lost in the matrix case. There is an intriguing comparison between the structure and the $\mathcal{S P \mathcal { R }}$ free parameter $G(z)$ in (3.14) and the Youla and Saito recursion. Likewise, the observation [17], that the scalar Nevanlinna algorithm coincides with the Cholesky decomposition of the Pick matrix, points towards a recursive solution, as does the association with Lagrange interpolation. In the matrix case, Blaschke products from scalar interpolation have a generalization in BlaschkePotopov products. A simple approach to the matrix interpolation problem using such products is given in [34]. The full extension remains a prospect for future work.

Another issue alluded to in [15] and reflected in earlier works is that the order of the interpolants can become very large. We note that the order of the interpolating solution is governed by the parameter $G(z)$ in Corollary 3.3. The formula (3.14) delivers the complete set of interpolants, although it is in no way clear how to find the minimal degree interpolant. A feature of the scalar circuit synthesis approaches is that it is frequently possible to establish that a particular realization is minimal, in terms of the number of required reactive elements for the synthesis. In the matrix case, the concepts of minimality persist and are tied to the degree of the state-variable realization, as in the scalar case. The prospect of reactance extraction or deflation procedures being ported from $n$-port circuit synthesis to the matrix interpolation problem in order to achieve an interpolant of manageable order also is a subject worthy of future consideration.

6. Conclusion. We have proven that a solution to Problem (W) always exists. That is, a finite set of sampled positive definite weighting function values from distinct frequencies suffices to determine a biproper $\mathcal{S P \mathcal { R }}$ transfer function matrix providing weighting function values at all frequencies, including infinity. The importance of this result is that it rules out the possibility of inadvertently introducing phase problems due to zeros or poles between samples, as commonly occurs in fitting digital filter coefficients using just the FFT. This should give some confidence to the application of these methods in determining the stability margin for MIMO control systems. Recent practice in the aerospace industries has seen the adoption of the minimal weighted generalized stability margin, described in Section 2, as the appropriate MIMO replacement for the gain and phase margin-based certification criteria for single-loop-closure plant-controller combinations of earlier years. Because of this linkage to practice, the results of this paper are necessary in providing confidence that the overall approach is sound.

There are a number of areas for development of these results. The most pressing would be to try to manage the order of the solutions as the number of interpolation points increases. It is clear that stable pole-zero cancellations are possible in $F(z)$ from (3.14), as is even clearer from iterative approaches based on Youla and Saito, where the cancellations occur on the imaginary axis by construction. However, it 
is not apparent how one might use the formulæ to search for low-order weighting functions. Certainly, the operator-theoretic connections between matrix interpolation and transfer function approximation suggest that some headway might be made in finding approximate low-order weighting functions.

Wherever the subject moves, it is clear that the linkages between control, circuits, and operator theory will be central. See [5] and especially the chapter from Young [44]. Brian Anderson's contributions will be evident throughout such an investigation.

\section{REFERENCES}

[1] J. Agler and J.E. McCarthy, Pick Interpolation and Hilbert Function Spaces, American Mathematical Society, Providence, RI, 2002.

[2] B.D.O. Anderson, Riccati Equations, Network Theory and Brune Synthesis: old solutions for contemporary problems, in Dynamical Systems Control, Coding, Computer Vision, G. Picci and D.S. Gilliam, eds., vol. 25 of Progress in Systems and Control Theory, Birkhäuser Verlag, 1999, pp. 1-25.

[3] B.D.O. Anderson and S. Vongpanitlerd, Network Analysis and Synthesis, Prentice-Hall, Englewood-Cliffs, NJ, 1973.

[4] Federal Aviation Authority, FAA Advisory Circular 33.28-1 June 2001, "Compliance Criteria for $14 C F R \S 33.28$, Aircraft Engines, Electrical and Electronic Engine Control Systems".

[5] S. Axler, J.E. McCarthy, and D. Sarason (eds), Holomorphic Spaces, vol. 33 of Mathematical Sciences Research Institute Publications, Cambridge University Press, Cambridge, UK, 1998.

[6] J.A. BALL, Interpolation problems of Pick-Nevanlinna and Loewner types for meromorphic matrix functions, Integral Equations and Operator Theory, 6 (1983), pp. 804-840.

[7] J.A. Ball, I. Gohberg, and L. Rodman, Interpolation of Rational Matrix Functions, vol. 45 of Operator Theory: Advances and Applications, Birkhäuser Verlag, Basel, 1990.

[8] C. BeCCARI, Interpolation on the $\omega$ axis, Electronics Letters, 4 (1968), pp. 139-140.

[9] V. Belevitch, Classical Network Theory, Holden-Day, San Francisco, CA, 1968.

[10] R.R. Bitmead and B.D.O. Anderson, Matrix fraction description of the lossless positive real property, IEEE Transactions on Circuits and Systems, 24 (1977), pp. 546-550.

[11] V. Bolotnikov And H. Dym, On boundary interpolation of matrix valued Schur functions, vol. 181 of Memoirs of the American Mathematical Society, American Mathematical Society, Providence, RI, 2006.

[12] S. Boyd and L. Vandenberghe, Convex Optimization, Cambridge University Press, 2004. also available at www.stanford.edu/ boyd/cvxbook.html.

[13] O. BRune, Synthesis of a finite two-terminal network whose driving point impedance is a prescribed function of frequency, Journal of Mathematical Physics, 10 (1931), pp. 191-236.

[14] E.W. Cheney, Introduction to Approximation Theory, Chelsea, New York, NY, USA, 1966.

[15] C.P. Coelho, J.R. Phillips, And L.M. Silveira, Passive constrained rational approximation algorithm using Nevanlinna-Pick interpolation, in Proceedings of the 2002 Design Automation and Test in Europe Conference and Exhibition (DATE'02), 2002, pp. 923-930.

[16] A. Dehghani, A. Lecchini-Visintini, A. Lanzon, and B.D.O. Anderson, Validating controllers for internal stability utilizing closed-loop data, IEEE Transactions on Automatic Control, 54 (2009), pp. 2719-2725.

[17] Ph. Delsarte, Y. Genin, and Y. Kamp, The Nevanlinna-Pick problem for matrix-valued functions, SIAM J. Appl. Math., 36 (1979), pp. 47-61. 
[18] P. Dorato, Analytic Feedback System Design: An Interpolation Approach, Brooks/Cole, Pacific Grove CA, 1999.

[19] P. Dorato, H-B. Park, And Y. LI, An algorithm for interpolation with units in $H^{\infty}$, with applications to feedback stabilization, Automatica, 25 (1989), pp. 427-430.

[20] J.C. Doyle, B.A. Francis, and A.R. Tannenbaum, Feedback Control Theory, MacMillan, New York, 1992.

[21] I.P. FedČInA, A criterion for the solvability of the Nevanlinna-Pick tangent problem, Math. Issled., 26 (1972), pp. 213-227.

$[22] —$ - A description of the solutions of the Nevanlinna-Pick tangent problem, Akad. Nauk Armjan. SSR Dokl., 60 (1975).

[23] E.A. Guillemin, Synthesis of Passive Networks, John Wiley \& Sons, New York, 1957.

[24] J. HARrison, Formal synthesis of circuits using linear matrix inequalities, in Proc. IEEE Int. Symp. Circuits Syst., vol. 1, 2005, pp. 232-235.

[25] A. Lanzon, Pointwise in frequency performance weight optimization in $\mu$-synthesis, International Journal of Robust and Nonlinear Control, 15 (2005), pp. 171-199.

[26] C. Mosquera and F. Perez, An algorithm for interpolation with positive rational functions on the imaginary axis, Automatica, 33 (1997), pp. 2277-2280.

[27] R. Nevanlinna, Über beschränkte Funktionen, die in gegebene Punkten vorgeschrieben Werte annehmen, Ann. Acad. Sci. Fenn., A13 (1919).

[28] — Über beschränkte analytische Funktionen, Ann. Acad. Sci. Fenn., A32 (1929), pp. 1-75.

[29] Y. Онта, H. Maeda, And S. Kodama, Unit interpolation in $H_{\infty}$ : bounds of norm and degree of interpolants, Systems \& Control Letters, 17 (1991), pp. 251-256.

[30] J. Park And R.R. Bitmead, Simultaneous scaling for MIMO controller certification, in Proceedings of the 46th IEEE Conference on Decision and Control, New Orleans, LA, 2007.

[31] V.V. Patel and K.B. Datta, A counter example for the conjecture in 'an algorithm for interpolation with units in $H_{\infty}$ ', Automatica, 31 (1995), p. 165.

[32] — - A note on direct interpolation algorithm for a strictly positive real function, IEEE Trans. Automatic Control, 40 (1995), pp. 1960-1962.

[33] G. PICK, Über die Beschränkungen analytischer Funktionen, welche durch vorgegebene Funktionswerte bewirkt werden, Math. Ann., 77 (1916), pp. 7-23.

[34] S.C. Power, An elementary approach to the matricial Nevanlinna-Pick interpolation criterion, Proceedings of the Edinburgh Mathematical Society, 32 (1989), pp. 121-126.

[35] R.K. Prasanth, Two-sided tangential interpolation with real rational units in $H_{\infty}$, Automatica, 34 (1998), pp. 861-874.

[36] D. Sarason, Nevanlinna-Pick interpolation with boundary data, Integral Equations and Operator Theory, 30 (1998), pp. 231-250.

[37] S. Skogestad and I. Postlethwaite, Multivariable Feedback Control, John Wiley \& Sons, New York, 1996.

[38] L.I. SMILEN, Interpolation on the real frequency axis, in IEEE International Convention Record, vol. 13 Part 7, 1965, pp. 42-50.

[39] G. Vinnicombe, Uncertainty and Feedback - $H_{\infty}$ loop-shaping and the $\nu$-gap metric, Imperial College Press, 2000.

[40] M. WOHLERs, Interpolation on the real frequency axis with positive-real or bounded-real functions, IEEE Transactions on Circuit Theory, 11 (1964), pp. 497-499.

[41] D.C. Youla, A new theory of cascade synthesis, IRE Transactions on Circuit Theory, 9 (1961), pp. 244-260.

[42] D.C. Youla, J.J. Bongionno JR, And C.N. Lu, Single-loop feeddback stabilization of linear multivariable dynamical plants, Automatica, 10 (1974), pp. 159-173.

[43] D.C. Youla And M. SAito, Interpolation with positive-real functions, Journal of the Franklin Institute, 284 (1967), pp. 77-108. 
[44] N.J. Young, Some function-theoretic issues in feedback stabilization, in Holomorphic Spaces, S. Axler, J.E. McCarthy, and D. Sarason, eds., vol. 33 of Mathematical Sciences Research Institute Publications, Cambridge University Press, Cambridge, UK, 1998, pp. 337-349.

[45] E. Zeheb And A. Lempel, Interpolation in the network sense, IEEE Trans Circuit Theory, 13 (1966), pp. 118-119.

[46] K. Zhou And J.C. Doyle, Essentials of Robust Control, Prentice Hall, New Jersey, 1998. 